Supplementary Material

\title{
Insight into the polymerization-induced self-assembly (PISA) via a realistic computer simulation strategy
}

Yu-Dou Yan, Yao-Hong Xue, Huan-Yu Zhao, Hong Liu*, Zhong-Yuan Lu, Feng-Long Gu

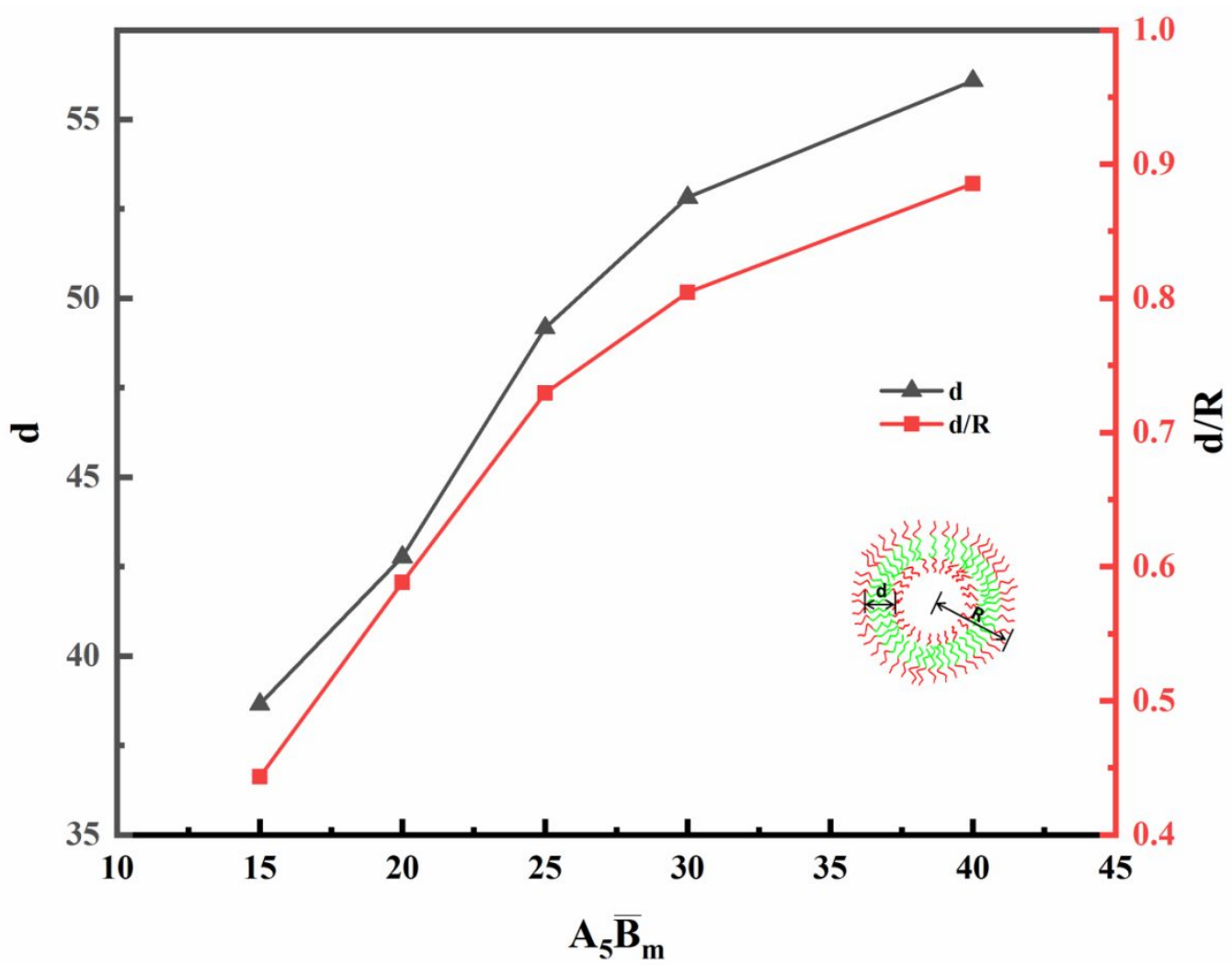

Figure S1. The dependence of the thickness of the vesicle $d$ (the distance from the outer shell to the inner chamber shell) and its ratio to the radius of the vesicle $\boldsymbol{d} R$ on the average length of the hydrophobic block $m$ in the system with $A_{5} \bar{B}_{m}$. 


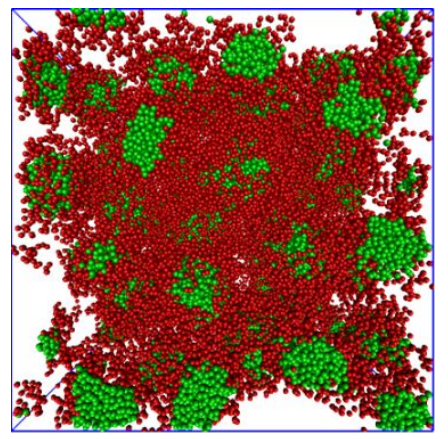

(a) $\mathrm{A}_{10} \mathrm{~B}_{10} \quad(f=20 \%)$

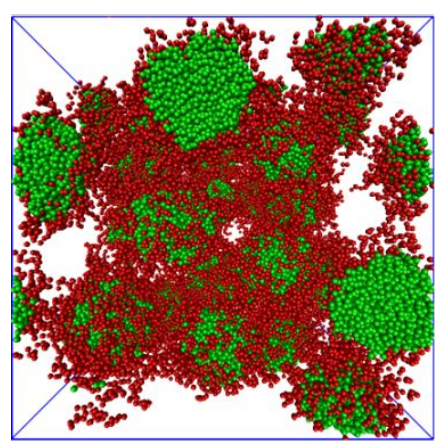

(c) $\mathrm{A}_{10} \mathrm{~B}_{20} \quad(f=20 \%)$

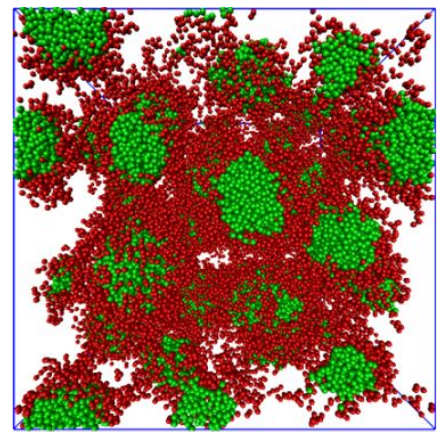

(b) $\mathrm{A}_{10} \mathrm{~B}_{15} \quad(f=\mathbf{2 0} \%)$

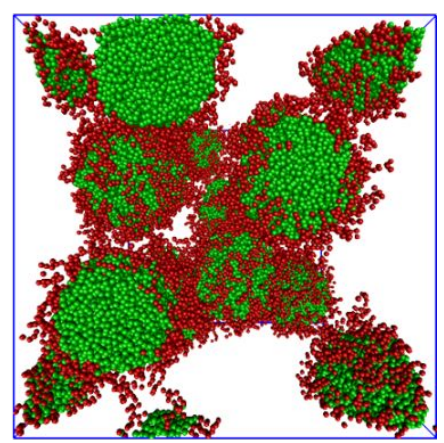

(d) $\mathrm{A}_{10} \mathrm{~B}_{40} \quad(f=\mathbf{2 0} \%)$

Figure S2. The typical final snapshots of the morphologies in PISA with the macro-CTA as $A_{10}$ but the feeding ratio is $A: B=1: 1 \sim 1: 4$. The free $B$ monomers and the solvent $S$ beads are omitted for clarity. The reaction probability is set as $P_{\mathrm{r}}=0.00005$ and the initial reactant concentration is set as $f=20 \%$. This figure typically shows the influence of hydrophobic block length with larger macro-CTA of $A_{10}$. 